\title{
Biodynamic imaging for phenotypic profiling of three-dimensional tissue culture
}

Hao Sun

Daniel Merrill

Ran An

John Turek

Daniela Matei

David D. Nolte 


\title{
Biodynamic imaging for phenotypic profiling of three-dimensional tissue culture
}

\author{
Hao Sun,, Daniel Merrill, ${ }^{a}$ Ran An,, ${ }^{\text {a }}$ John Turek, ${ }^{c}$ Daniela Matei, ${ }^{d}$ and David D. Nolte ${ }^{a, *}$ \\ apurdue University, Department of Physics, 525 Northwestern Avenue, West Lafayette, Indiana 47907, United States \\ ${ }^{\mathrm{b}}$ Animated Dynamics, Inc., 5770 Decatur Boulevard Suite A, Indianapolis, Indiana 46241, United States \\ 'Purdue University, Department of Basic Medical Sciences, West Lafayette, 625 Harrison Street, Indiana 47907, United States

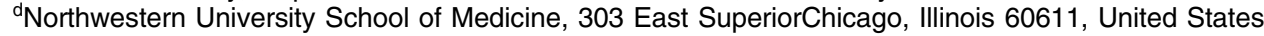

\begin{abstract}
Three-dimensional (3-D) tissue culture represents a more biologically relevant environment for testing new drugs compared to conventional two-dimensional cancer cell culture models. Biodynamic imaging is a highcontent 3-D optical imaging technology based on low-coherence interferometry and digital holography that uses dynamic speckle as high-content image contrast to probe deep inside 3-D tissue. Speckle contrast is shown to be a scaling function of the acquisition time relative to the persistence time of intracellular transport and hence provides a measure of cellular activity. Cellular responses of 3-D multicellular spheroids to paclitaxel are compared among three different growth techniques: rotating bioreactor (BR), hanging-drop (HD), and nonadherent (U-bottom, UB) plate spheroids, compared with ex vivo living tissues. HD spheroids have the most homogeneous tissue, whereas BR spheroids display large sample-to-sample variability as well as spatial heterogeneity. The responses of BR-grown tumor spheroids to paclitaxel are more similar to those of ex vivo biopsies than the responses of spheroids grown using HD or plate methods. The rate of mitosis inhibition by application of taxol is measured through tissue dynamics spectroscopic imaging, demonstrating the ability to monitor antimitotic chemotherapy. These results illustrate the potential use of low-coherence digital holography for 3-D pharmaceutical screening applications. ๑ 2017 Society of Photo-Optical Instrumentation Engineers (SPIE) [DOI: 10 .1117/1.JBO.22.1.016007]
\end{abstract}

Keywords: three-dimensional tissue growth; biodynamic imaging; digital holography; low-coherence interferometry; cellular dynamics; dynamic light scattering; speckle; biomedical imaging; drug development; drug discovery; pharmaceutical; biological relevance; high-content imaging; physiological effects of paclitaxel.

Paper 160636R received Sep. 12, 2016; accepted for publication Nov. 28, 2016; published online Jan. 9, 2017.

\section{Introduction}

Drug screening for pharmaceutical development has relied on two-dimensional (2-D) cell culture because of the ease of imaging thin layers of cells using conventional as well as advanced forms of microscopy. However, the 2-D environment is not representative of biological tissue, as cells live in a threedimensional (3-D) microenvironment with physical as well as chemical contacts to their local environment. 3-D cell culture systems overcome many of the limitations of traditional 2-D monolayer cell culture systems by mimicking more closely the complex cellular heterogeneity and tumor microenvironmental conditions. ${ }^{1}$ In addition, it is recognized that cellular dimensionality affects cancer drug sensitivity or resistance in 3 -D cultures relative to 2-D monolayers. ${ }^{2}$ Consequently, there is a growing trend toward 3-D culture to capture more biologically relevant tissue response to applied compounds. ${ }^{3-9}$ However, deep-tissue optical imaging techniques capable of measuring pharmaceutical effects inside living tissue are currently lacking. Candidate imaging approaches include confocal and nonlinear microscopy, ${ }^{10}$ light-sheet microscopy, ${ }^{11}$ and optical coherence tomography (OCT). ${ }^{12,13}$ Of these, only OCT has significant depth penetration (millimeters) to move deeper than surface layers of cells. OCT has been used to image pharmaceutical responses of 3-D tissues, ${ }^{14-17}$ but it lacks high-content screening capabilities that are essential to characterize complex pharmaceutical response of tissue.
Biodynamic imaging (BDI) ${ }^{18-20}$ is an optical imaging technology related to en face $\mathrm{OCT},{ }^{21}$ but with enhanced partially coherent speckle generated by broad-area illumination with coherence detection through digital holography. ${ }^{22-24}$ BDI penetrates up to $1 \mathrm{~mm}$ into living tissue and returns high-content information in the form of dynamic light scattering across a broad spectral range. ${ }^{25,26}$ The fluctuation frequencies relate to Doppler frequency shifts caused by light scattering from the subcellular constituents that are in motion. The speeds of intracellular dynamics range across four orders of magnitude from tens of nanometers per second (cell membrane $)^{27-30}$ to tens of microns per second (organelles, vesicles). ${ }^{31-34}$ In the nearinfrared backscattering geometry, these speeds correspond to Doppler frequencies from 0.005 to $50 \mathrm{~Hz}$.

Frequency-domain decomposition of the light fluctuations using tissue dynamics spectroscopy ${ }^{19}$ produces broad-band fluctuation spectra that encode the wide variety of subcellular motions. When pharmaceutical compounds are applied to the tissue, dynamic cellular processes are modified, and these modifications appear as changes in the fluctuation spectra. By applying reference compounds with known mechanisms of action, a library of drug-response spectrograms can be generated against which drug screens may be compared, gaining information about the effect of the compound on cellular processes such as necrosis and apoptosis. ${ }^{35}$ This type of phenomenological assay is known as a phenotypic profile. Phenotypic profiling 
has seen a resurgence in recent years as a more systems-based approach to drug discovery and development ${ }^{36}$ that captures the complex interplay of cellular processes affected by the drug candidate. Phenotypic profiling of dynamics using digital holography on 2-D culture has been performed, ${ }^{37}$ but does not probe the 3-D microenvironment of tissues.

This paper describes the application of BDI for the phenotypic profiling of responses to chemotherapy using distinct 3-D tissue culture approaches. Our results demonstrate that different culture approaches, even when applied to the same cancer cell line, produce tissues with distinct cellular sensing of chemotherapy, as measured by BDI.

\section{Materials and Methods}

Several methods are used to generate 3-D multicellular tumor spheroids including: (i) Bioreactors(BRs) in which cells or spheroids aggregate in the chamber; ${ }^{38-40}$ (ii) nonadherent plates that prevent attachment; ${ }^{41,42}$ (iii) hanging-drop (HD) plates where cells are grown in a suspended droplet to form one single spheroid; ${ }^{4-46}$ (iv) multicellular layers; ${ }^{47-49}$ and (v) organoid culture $^{50,51}$ among others. In this paper, BDI was used to assess the different cellular responses to paclitaxel of cancer spheroids obtained by using the three most common approaches: BR, HD, and nonadherent plate growth. Within these growth approaches, there are important differences that may relate to the downstream success rate for drug screening and development.

\subsection{Three-Dimensional Tissue Culture}

Three different multicellular spheroid tissue growth methods were used for 3-D tissue culture: (1) a Synthecon (Houston, Texas) rotating BR, (2) Corning (Corning, New York) U-bottom (UB) spheroid plates, and (3) 3D Biomatrix (Ann Arbor, Michigan) HD plates. Two cell lines, A2780 (human ovarian cancer) and DLD-1 (human colon adenocarcinoma), were obtained from the American Type Culture Collection (Manassas, Virginia) and grown at $37^{\circ} \mathrm{C}$ in a humidified $5 \% \mathrm{CO}_{2}$ incubator in RPMI-1640 medium supplemented with $10 \%$ fetal bovine serum (Atlanta Biologicals, Lawrenceville, Georgia), $100 \mathrm{U} / \mathrm{mL}$ of penicillin, and $100 \mu \mathrm{g} / \mathrm{mL}$ of streptomycin (Sigma). To grow spheroids in the BR, the cells from a $25-\mathrm{cm}^{2}$ flask $\left(\sim 2 \times 10^{6}\right.$ cells) were placed in a 50-mL capacity rotating drum BR in complete growth medium. The medium was refreshed $(30 \%)$ every other day until the cells formed spheroids of optimal experimental size (300 to $800 \mu \mathrm{m}$ in diameter). Cells were seeded at a concentration of 5000/well in Corning spheroid plates and 5000/drop in the 3D Biomatrix HD plate. Spheroids formed within 24 to $48 \mathrm{~h}$ in these plate-based methods.

\subsection{Ex Vivo Tumor Explants}

Established cancer cell lines and xenografts have been the mainstay of cancer research for the past several decades. To date, the analysis of drug sensitivity and resistance in both established cancer cell lines and xenografts has been indispensable. However, while testing of compounds in established cancer cell lines is often not predictive of clinical efficacy, corresponding in vivo studies in animals are very expensive and time consuming. Therefore, heightened attention is being paid toward ex vivo analysis of tumor explants due to the greater understanding that cancer therapeutic response is not exclusive to the inherent molecular composition of cancer cells but rather is greatly influenced by the tumor cell microenvironment, a feature that cannot be recapitulated by traditional culturing methods. Ex vivo treatment response analysis could become a standard tool in preclinical and clinical development of cancer therapeutics and is envisioned as a step toward a personalized medicine approach for new therapeutic development strategies. ${ }^{52,53}$

Tumor xenografts were generated in nude mice using human ovarian carcinoma cell lines (A2780, SKOV-3). Animal procedures were approved by the Indiana University School of Medicine Animal Care and Use Committee and were in accordance with federal regulations. Female nude mice (nu/nu BALB/ c) 7- to 8-weeks old (Harlan, Indianapolis, IN) were subcutaneously injected in the right flank with $2 \times 10^{6}$ cells or orthotopically injected in one of the ovaries. The mice were euthanized 2 to 4 weeks after the injection and the tumor xenografts removed. Tumor xenografts were diced into $1 \mathrm{~mm}^{3}$ pieces and immobilized in 96-well cell culture plates in a thin layer of $1 \%$ low gel-temperature agarose made up in HEPES buffered RPMI 1640 medium (ATCC, Manassas, Virginia).

\subsection{Biodynamic Imaging}

BDI was performed with an optical instrument that captures backscattered light from biological samples and records digital holograms on a CCD Fourier-plane chip. The light source is a continuous-wave low-coherence light source (Superlum, Cork, Ireland) with a $20-\mathrm{mW}$ output intensity at a wavelength of $850 \mathrm{~nm}$ and a bandwidth of $15 \mathrm{~nm}$ with a coherence length of $\sim 15 \mu \mathrm{m}$. The optical section thickness is significantly broadened by multiple scattering with increasing broadening with increasing gated depth. The BDI system is a Mach-Zehnder interferometer shown in Fig. 1. The light path is divided into a signal and reference arm by a polarizing beam splitter with a variable polarizer to adjust the relative intensities in the signal and reference arms. Dynamic light scattering is performed in a back-scatter geometry because the sensitivity to Doppler frequency shifts depends on the momentum transfer vector that selects longitudinal motion along the backscatter direction. The maximum backscattering momentum transfer is given by $q=4 \pi n / \lambda$, where $n$ is the refractive index of the tissue and $\lambda$ is the free-space wavelength. The reduced wavelength inside the medium is given by

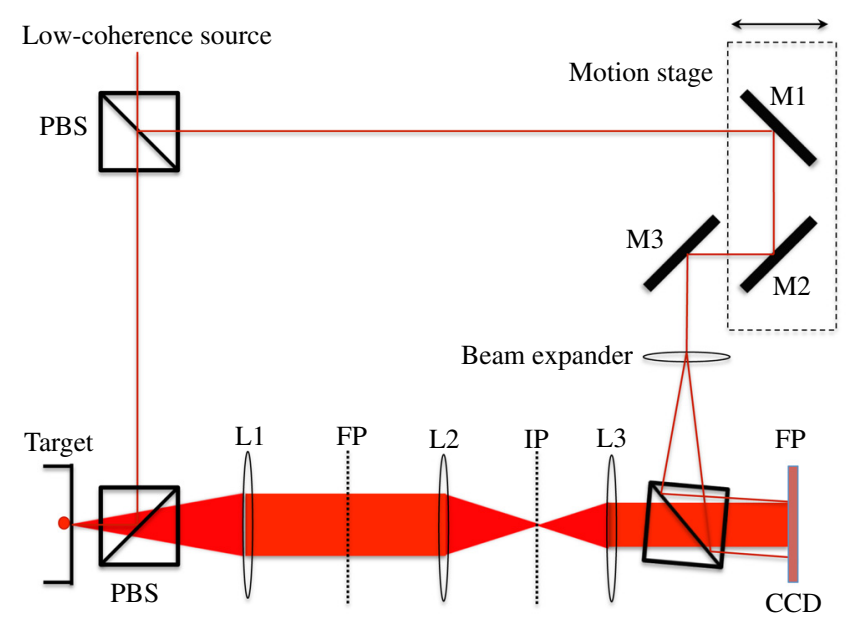

Fig. 1 Fourier domain low-coherence digital holography system with a Mach-Zehnder off-axis configuration recording on the FP. The object light backscattered by the target is collected and projected onto the FP at the CCD by lenses L1, L2, and L3 with intermediate image plane and FP. 


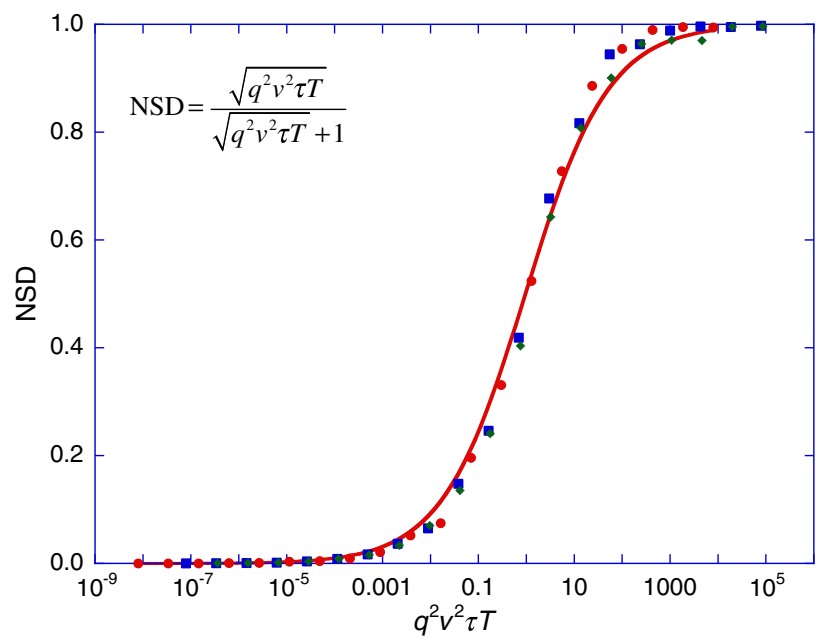

Fig. 2 NSD as a function of the scaling parameter $q^{2} v^{2} \tau T$, where $q$ is the momentum transfer, $v$ is the organelle speed, and $\tau$ is the persistence time of the random flights. Monte Carlo simulation of flights were performed with 500 frames at $25 \mathrm{fps}$ for $T=20 \mathrm{~s}$. The NSD data are accumulated from simulations using different organelle velocities and persistence times, demonstrating the scaling relationship between NSD and the transport properties. For $q^{2} v^{2} \tau T \gg 1$, the NSD approaches unity.

$\lambda_{\text {red }}=\lambda_{\text {free }} / 4 \pi n$. Assuming a refractive index $n=1.4$ and a freespace wavelength of $840 \mathrm{~nm}$, the reduced wavelength is equal to $50 \mathrm{~nm}$. The light scattered by the living biological sample is collected by a long focal-length lens and transformed to a Fourier plane (FP) where the CCD pixel array is placed. The reference wave is incident on the CCD array at a small angle of 3 deg relative to the signal axis, creating an off-axis digital hologram with a fringe spacing of $25 \mu \mathrm{m}$ on the camera chip. A digital hologram is acquired on the FP of the optical imaging system, and this Fourier-domain hologram is transformed using an FFT algorithm into the image domain. The transformation performs two functions: (1) demodulating the spatial carrier frequency represented by the holographic interference fringes and (2) coherence-gating the low-coherence light to a specified depth inside the tissue sample.

The coherence-gating role of digital holography creates a full-frame OCT section of the tumor spheroid at a fixed depth. The reconstructed section is highly speckled, which normally is considered to be an undesirable side effect in OCT, but dynamic speckle for our application provides the basis for BDI. BDI captures successive frames at a fixed coherence gate at a frame rate of $25 \mathrm{~Hz}$. In dynamic light scattering, there are two limits related to the characteristic fluctuation frequency depending on the mean speeds of intracellular motions. The Doppler limit corresponds to mean free flight distances longer than the reduced wavelength, for $q v \tau \gg 1$, where $v$ is the intracellular speed and $\tau$ is the persistence time for the transport. The opposite limit is the diffusion limit when $q v \tau \ll 1$. In this limit, the diffusion is still dominated by random flights rather than thermally driven Brownian motion, but the transport is a random walk, driven by active transport such as molecular motors or cytoskeletal restructuring. The diffusion coefficient in this limit is given by $D=v^{2} \tau$, and the diffusion and Doppler angular frequencies, in the respective limits, are

$\omega_{\text {Diff }}=q^{2} D, \quad \omega_{\text {Dop }}=q v$.

Living tissue is highly complex, with a wide range of speeds and persistence times. The measured knee frequency (half-width half-maximum of the spectrum) for the fluctuation spectrum scales as

$\omega_{\text {knee }}=\frac{\omega_{\text {Dopp }}^{2} \tau}{1+\omega_{\text {Dop }} \tau}=\frac{q^{2} v^{2} \tau}{1+q v \tau}$,

which is equal to $\omega_{\text {Dop }}$ for $q v \tau \gg 1$ and is equal to $\omega_{\text {Diff }}$ for short $q v \tau \ll 1$. The experimentally measured knee frequencies for 3 -D tissue growth as well as for ex vivo biopsies are in the range from 0.01 to $0.1 \mathrm{~Hz}$ corresponding to intracellular speeds from 3 to $200 \mathrm{~nm} / \mathrm{s}$. Speeds on the order of $3 \mathrm{~nm} / \mathrm{s}$ are associated with cell shape changes as cells respond to their force environment. ${ }^{54-57}$ Speeds on the order of $30 \mathrm{~nm} / \mathrm{s}$ are associated with nuclear motions and membrane dynamics. ${ }^{28,33,58}$ Speeds on
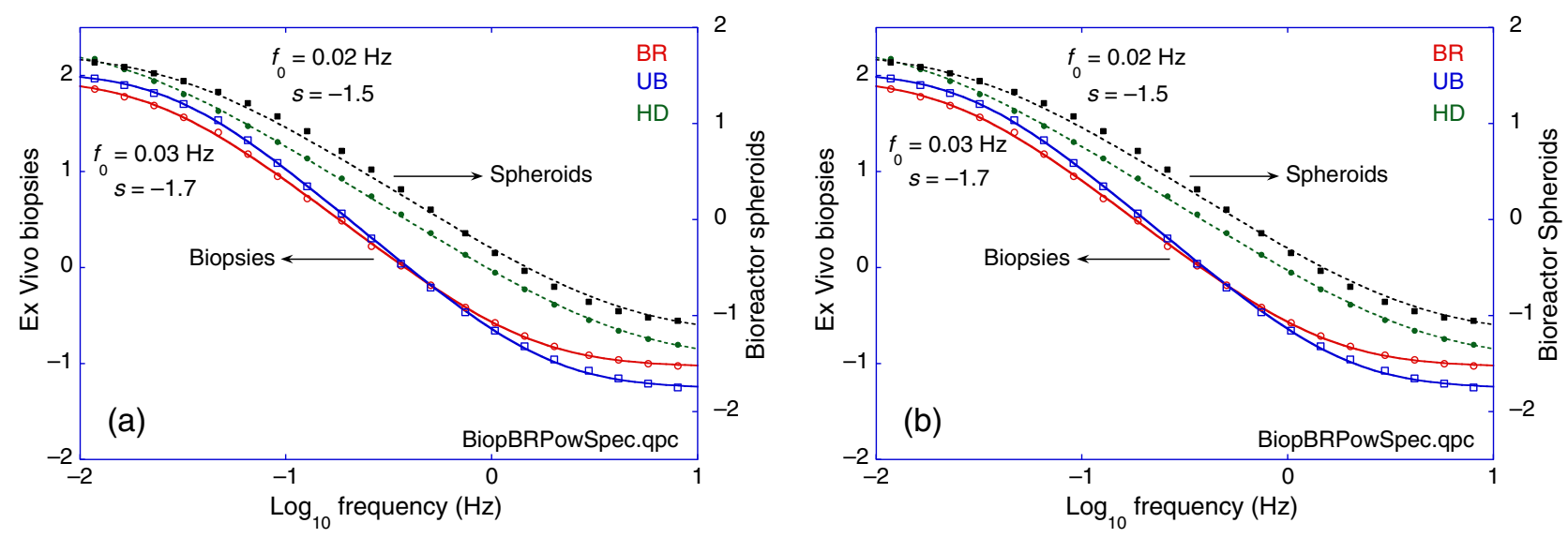

Fig. 3 (a) The measured fluctuation power spectra of A2780 and DLD-1 spheroids for three growth techniques: $\mathrm{BR}$, bioreactor (red); UB, U-bottom (blue); and HD, hanging drop (green). The data are plotted on double $\log _{10}$. The knee frequencies are in the range of 0.02 to $0.03 \mathrm{~Hz}$. The slope parameters are around -1.5 to -1.7 , and the dynamic range is $\sim 1000: 1$. The tumors from the BR have lower knee frequencies than tumors from the HD method. (b) Mouse (red) and human esophageal biopsies (blue) have lower dynamic range relative to the spectra of A2780 (green) and DLD-1 (black) tumor spheroids obtained from the BR. 
the order of $300 \mathrm{~nm} / \mathrm{s}$ and above are associated with organelle transport and cytoskeletal restructuring. ${ }^{59-61}$

\subsection{Finite-Sampling Effects}

A common metric that characterizes tissue health is the normalized standard deviation (NSD), also known as temporal speckle contrast, ${ }^{62}$ that is calculated from the standard deviation of the intensity divided by the average intensity. Large fluctuations in the dynamic speckle images result in high NSD values, and a 2-D map of NSD values gives a visual measure of motility, called motility contrast imaging (MCI). The MCI map displays the NSD from a biological sample as a representation of the activity/health of the sample. A high NSD can indicate vitality of the sample, high proliferation, or it may represent a high overall metabolic rate. For example, 3-D spheroids of known aggressive cancer cell lines with short doubling times and high metabolism typically have a higher NSD value than less aggressive cancers for spheroids of equivalent health. For an exponential probability distribution function of intensities, the standard deviation is equal to the mean, and therefore under ideal sampling NSD $=1$. However, for finite sampling, the NSD is less than unity and depends on the finite-sampling time as well as on the characteristic persistence times of the subcellular motion. Figure 2 shows the NSD versus the scaling quantity $q^{2} v^{2} \tau T$ for a set of 100 Monte Carlo simulations using random particle flights with speeds of 0.1 and $1 \mu \mathrm{m} / \mathrm{s}$ with persistence times of 0.1 and $1 \mathrm{~s}$. The time $T$ is the total acquisition time, and $\tau$ is the persistence time. The NSD approaches 1 as $q^{2} v^{2} \tau T \gg 1$ and NSD changes rapidly when $q^{2} v^{2} \tau T \sim 1$. The condition for $\mathrm{NSD}=0.5$ is $q^{2} v^{2} \tau T=1$. Because $q$ and $T$ are fixed parameters of the experiment, changes in NSD induced by applied therapies are caused by changes in the $v^{2} \tau$ product, capturing the influence of the therapy on the physiological motions of
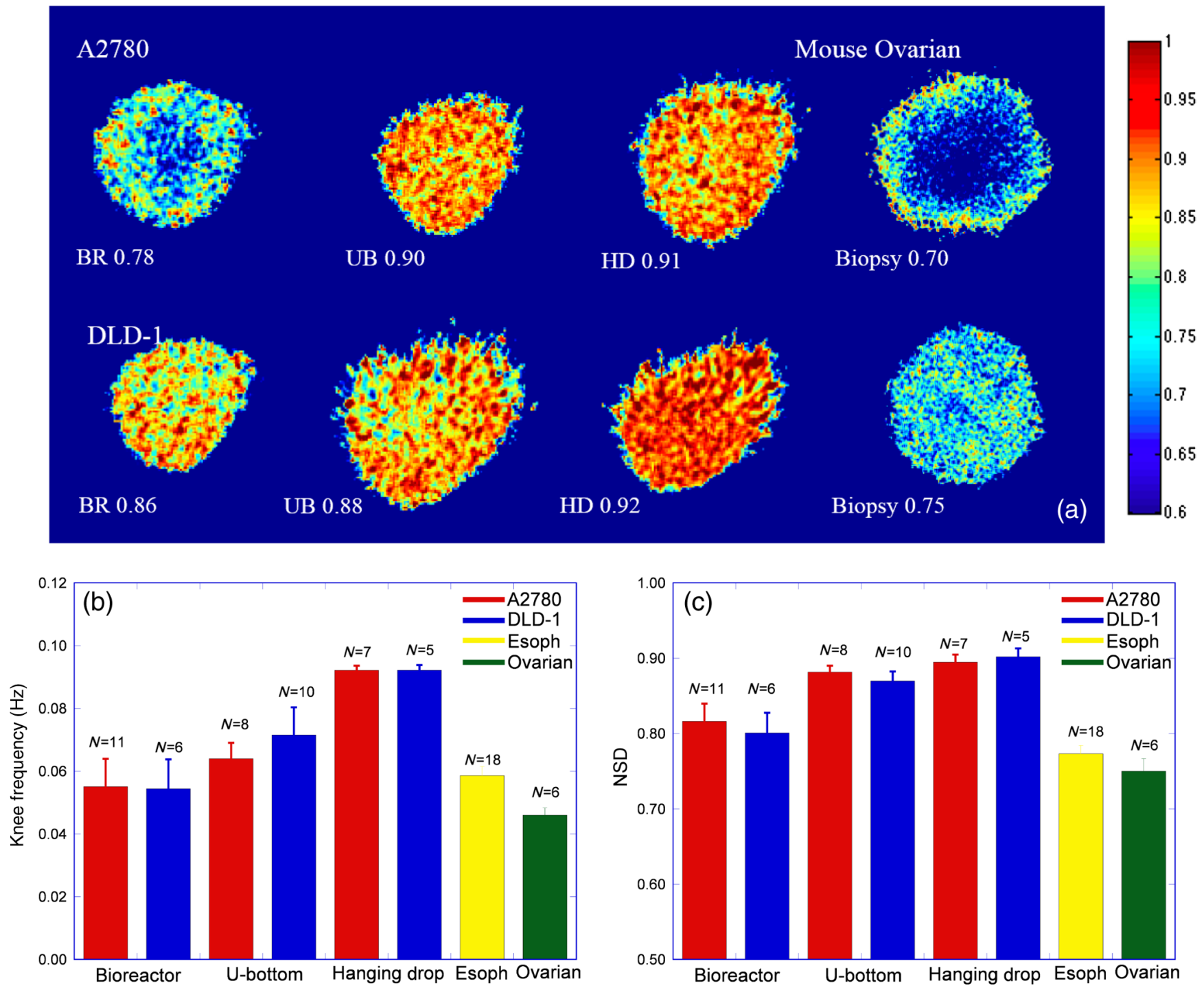

Fig. 4 (a) MCl of A2780 and DLD-1 spheroids for three different growth methods: BR, bioreactor; UB, U-bottom; and HD, hanging-drop, and compared with ovarian tumor xenografts. The color scale ranges from 0.6 to 1. The BR spheroids have lower NSD in the core area because the BR spheroids are tighter and denser. UB and HD spheroids have higher NSD because of lower adhesions. (b) Bar chart of knee frequency and (c) NSD of spheroids and biopsies. BR spheroids have a large variability. HD spheroids have the most uniform properties. 
the living sample. The common behavior for different velocities and persistence times is described by the relationship

$$
\mathrm{NSD}=\frac{\sqrt{q^{2} v^{2} \tau T}}{\sqrt{q^{2} v^{2} \tau T}+1},
$$

which demonstrates the finite-time sampling effects on the NSD. This expression relates to a common speed and persistence time among all scatterers, whereas in biological samples there is a distribution of speeds and persistence times.

The time traces of the fluctuating intensities are transformed into the frequency domain as a spectral power density denoted by $S(\omega)$. The measured power spectrum is a function of the frame rate of the acquisition and exposure time of the shutter. In particular, the Nyquist theorem states that the highest frequency that can be resolved is $f_{\max }=\mathrm{fps} / 2$, where fps stands for frames per second. In addition, during the data acquisition, the detection bandwidth is set by the exposure time as $f_{\mathrm{BW}}=$ $1 /\left(2 t_{\exp }\right)$, where $t_{\exp }$ is the exposure time that "freeze-frames" motion, like a strobe, between the Nyquist frequency and the detection bandwidth. The integral of the spectral power between the Nyquist frequency and the detection bandwidth appears as a baseline at the Nyquist frequency. This is called the Nyquist floor and is not, in general, a noise floor but contains information about fast vesicle motions with frequencies up to $f_{\mathrm{BW}}$. The spectrum also has a noise floor that can contribute to the integral, but this contribution is usually small relative to the Nyquist floor in active tissues.

\section{Experimental Results}

\subsection{Biodynamic Growth Characterization}

A selection of representative power spectra is shown in Fig. 3 for $\mathrm{BR}, \mathrm{HD}$, and UB plate spheroids as well as living biopsies. In Fig. 3(a), the HD spheroids have the highest knee frequency because they are more active than the other two growth methods. Figure 3(b) shows the spectra of a mouse ovarian xenograft biopsy and a human esophageal biopsy compared with tumor spheroids from a BR. The NSD of the tumor biopsies have mean values around 0.8 , which are similar to values for the
BR sample, and the power spectra have similar knee frequencies around $0.05 \mathrm{~Hz}$. The similarity between the biopsies and the BR tumor spheroids provides support for the conclusion that the BR sample performance is closer to the biopsy than the HD and UB methods. This could reflect the longer culture period in the $\mathrm{BR}$, enabling the formation of extracellular matrix (ECM), and denser cellular adhesions, ${ }^{63}$ rendering BR-grown spheroids more similar to in vivo grown tumors.

Motility contrast images (MCI) of the A2780 and DLD-1 cell lines under the three growth methods are shown in Fig. 4(a). The NSD increases from the BR to UB to HD for each cell line. An averaged NSD of 0.79 for BR DLD and 0.82 for BR A2780, and a higher NSD of 0.9 for the HD DLD and A2780 spheroids were measured. The BR usually takes several weeks to grow tumor spheroids to the optimal size, which allows it to develop more ECM and complex cellular adhesions than the other methods. ${ }^{64}$ Conversely, the UB and HD methods take 1 to 2 days to accomplish the growth, and hence they tend to be looser aggregates of cells. It is typical for the BR spheroids to have low motility in the core of the spheroid because the cells in the core area have been exposed to lower oxygen and nutrients for a longer period of time, which can be seen from the MCI of the BR-grown A2780 spheroid in Fig. 4(a). Unlike the BR spheroids, the UB and HD spheroids tend to be more uniform because the cells are less dense, and nutrients can be transported more easily from the outer environment into the spheroids. ${ }^{49,65-67}$

The average NSD values and knee frequencies are shown in Figs. 4(b) and 4(c). The sample numbers for these data are A2780: BR $N=11$, UB $N=8$, and HD $N=7$; DLD1: BR $N=6$, UB $N=10$, and HD $N=5$. The BR method has the smallest average NSD and knee frequencies, and the HD method has the highest average NSD values and knee frequencies. On the other hand, the BR samples have large sample-to-sample variability with varying spheroid metabolic and physiological activity. The variation is diverse because of the long duration of the BR growth. In the same batch, some spheroids may be actively growing, while others may be dying out. The HD method produces spheroids with higher NSD and knee frequencies with smaller variability. Figures 5(a) and 5(b) show the
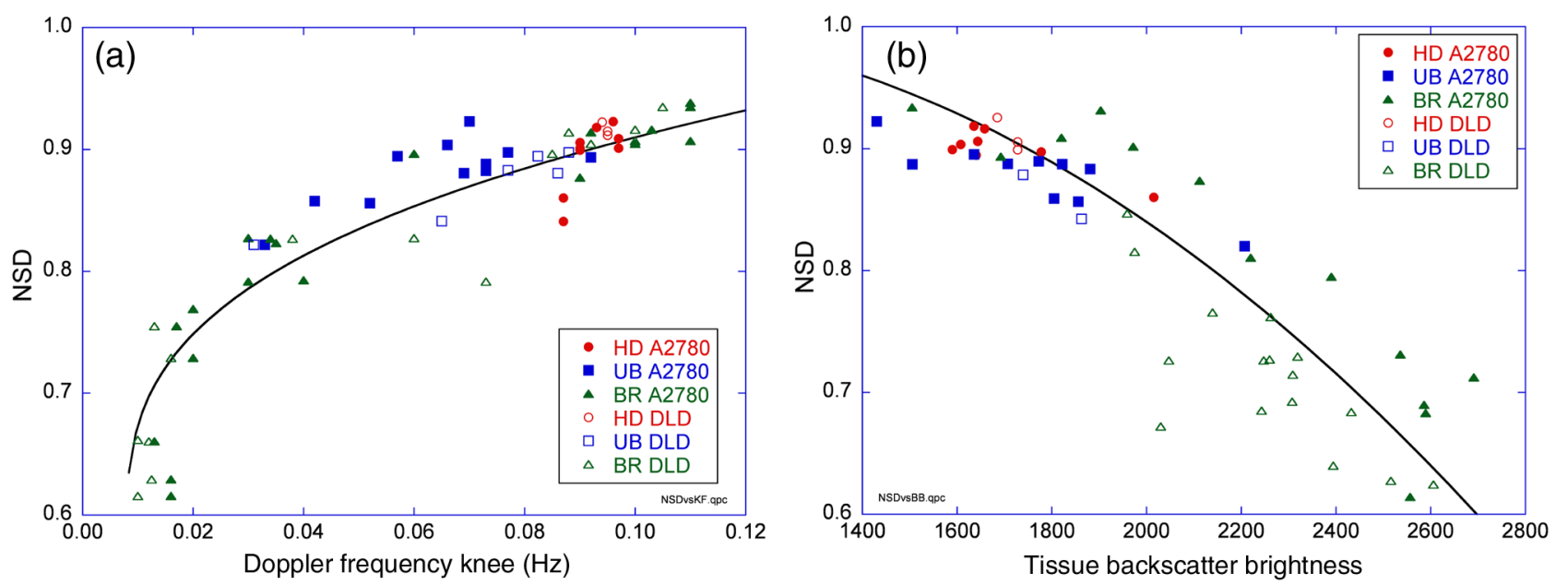

Fig. 5 NSD correlated against other properties of the tissue. (a) NSD versus knee frequency of the fluctuation power spectrum for A2780 and DLD-1 spheroids. (b) NSD versus BB for A2780 and DLD-1 spheroids. The BR samples have higher BB because they are more optically heterogeneous. UB and HD spheroids are more transparent. The HD method produces the most uniform tumor spheroid properties. 
relation between NSD versus knee frequency and NSD versus backscattering brightness. The HD and UB growth techniques produce homogeneous spheroids, and the knee frequency and NSD tend to be high. Unlike HD and UB growth techniques, the BR growth technique produces spheroids with a wide variation. In Fig. 5(a), the NSD and knee frequency vary from 0.6 to 0.9 , and 0.01 to 0.1 , respectively. A similar trend occurs in the backscattering brightness in Fig. 5(b). Spheroids from HD and UB plates have low backscattering brightness. BR spheroids have higher backscattering brightness because the BR spheroids are structurally heterogeneous. Spheroids from HD and UB techniques are more uniform, but have low ECM and low adhesion density. Therefore, HD and UB plates are good for high-viability and high-uniformity requirements in drug screening, whereas the BR growth is more suitable for experiments that are dependent upon more developed ECM and cell junctions.

(a)
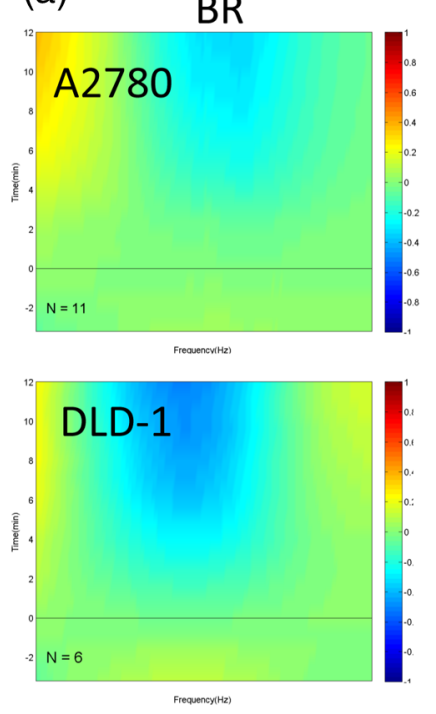

UB
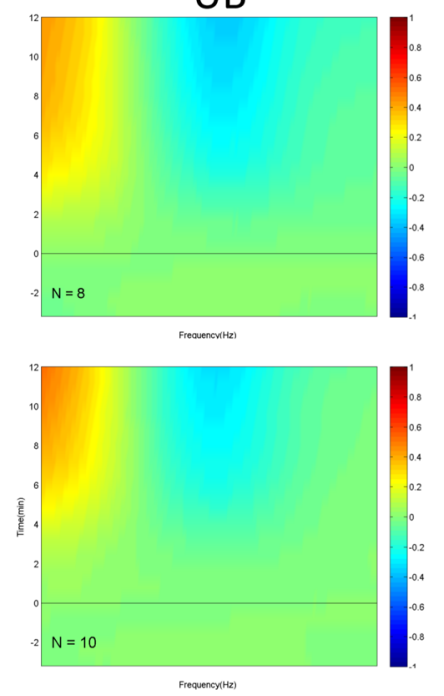

\subsection{Biodynamic Drug Response}

When a drug is applied to a sample, or the environmental conditions change, the relative power density at different frequencies is altered. This change is captured through the differential relative spectral power density defined as

$$
D(\omega, t)=\log [S(\omega, t)]-\log \left[S\left(\omega, t_{0}\right)\right],
$$

where $S(\omega, t)$ is the power spectrum at time $t$ and $S\left(\omega, t_{0}\right)$ is the baseline prior to the perturbation of the tissue. Paclitaxel, a cytoskeletal drug, is used as a gold standard reference compound to measure the relative spectral power density change because it stabilizes the microtubule polymers and stops cell division. ${ }^{63,68}$ Paclitaxel prolongs activation of the mitotic checkpoint, triggering apoptosis, or reversion to the G-phase of the cell cycle without cell division. Paclitaxel was added into the

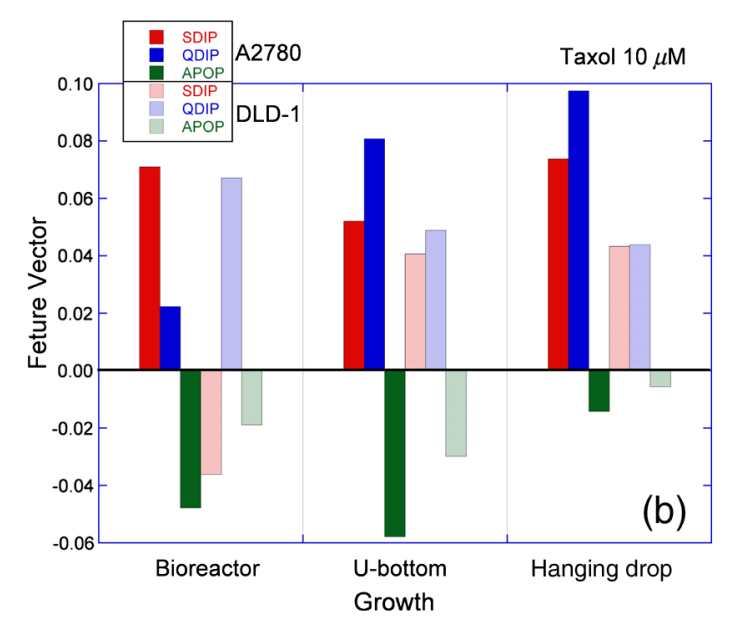

Spectogram format (time-frequency)

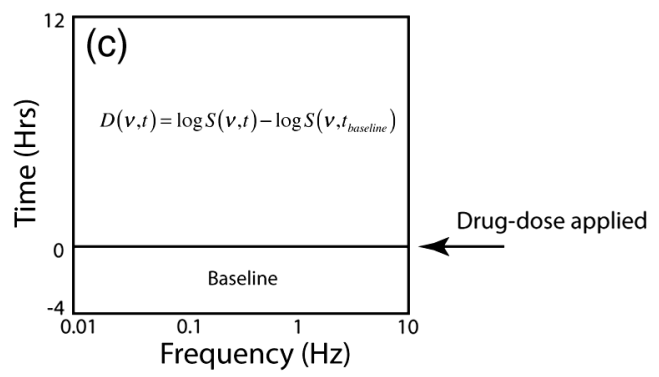

Fig. 6 (a) Paclitaxel time-frequency dose-response averaged spectrograms of A2780 and DLD-1 for three growth methods (BR, bioreactor; UB, U-bottom plate; and HD, hanging drop). The 4-h baseline is under the control medium (DMSO carrier), and $10 \mu \mathrm{M}$ paclitaxel was added at $t_{0}=0$. Red and blue colors correspond to increase or decrease in the differential power density, respectively. The response of the paclitaxel shows mid-frequency suppression from inhibited mitosis. The right-most graphs are the averaged responses from control media. (b) Bar chart of three biodynamic biomarkers: QDIP, SDIP, and APOP that capture symmetric and asymmetric frequency patterns, respectively, and apoptotic behavior. (c) Format for the time-frequency spectrograms. 
growth medium at time $t_{0}$ at a concentration of $10 \mu \mathrm{M}$. In Fig. 6 (a), the spectrograms show the relative spectral density increase/ decrease in response to the drug. The frequency axis is logarithmic and extends from 0.01 to $12.5 \mathrm{~Hz}$. The time axis extends from -4 to $12 \mathrm{~h}$, in which the application of the dose is made at $t_{0}=0$. There are $4 \mathrm{~h}$ of baseline and $12 \mathrm{~h}$ of dose response. The A2780 and DLD-1 spheroids have similar responses to the paclitaxel, with strong enhancement in the low frequency $(<0.1 \mathrm{~Hz})$ band and inhibited mid frequency $(0.1$ to $1 \mathrm{~Hz})$.

The drug-response spectrograms exhibit recognizable features that occur in characteristic frequency ranges at characteristic times after a dose is applied. There are many ways that the time-frequency plane can be partitioned and quantified into a feature vector. For the spectrograms in Fig. 6(a), we quantified three feature metrics called SDIP, QDIP, and APOP. The two features SDIP and QDIP are obtained by multiplying the spectrograms by linear masks that represent red shifted (spectral weight moving to lower frequencies) and mid-enhanced (increased spectral weight in central frequencies, and decreased spectral weight at high and low frequencies) frequency patterns, respectively. The feature APOP is a nonlinear mask that measures combined enhanced low frequencies and enhanced high frequencies that captures organelle transport and blebbing processes during apoptosis. ${ }^{58}$
The three biodynamic biomarkers are shown in Fig. 6(b) for the two cell lines and three growth methods. The biomarkers are consistent between the A2780 and DLD-1 cell lines for the HD and UB growth. However, the SDIP biomarker for the BR-grown samples have different signs between the two cell lines. Here again the HD and UB techniques show better homogeneity and similarities between these growth techniques, but the BR samples are less consistent. This is an important conclusion for researchers seeking to perform consistent drug screens in 3-D tissue.

\section{Pharmacodynamics}

Drug sensitivity can depend on adhesion density through the interplay between adhesions and the cytoskeleton that is affected by paclitaxel. Adhesion density also depends on the growth method. ${ }^{60}$ Two consistent metrics that can be tracked in time are the brightness of the samples and the temporal speckle contrast (NSD). Temporal speckle contrast is a direct measure of overall activity, and backscatter brightness (BB) increases with increasing optical heterogeneity, which parallels cellular structural heterogeneity. The time-dependences of NSD and BB are shown in Fig. 7. The application of paclitaxel decreases the temporal speckle contrast for all cell line and growth cases
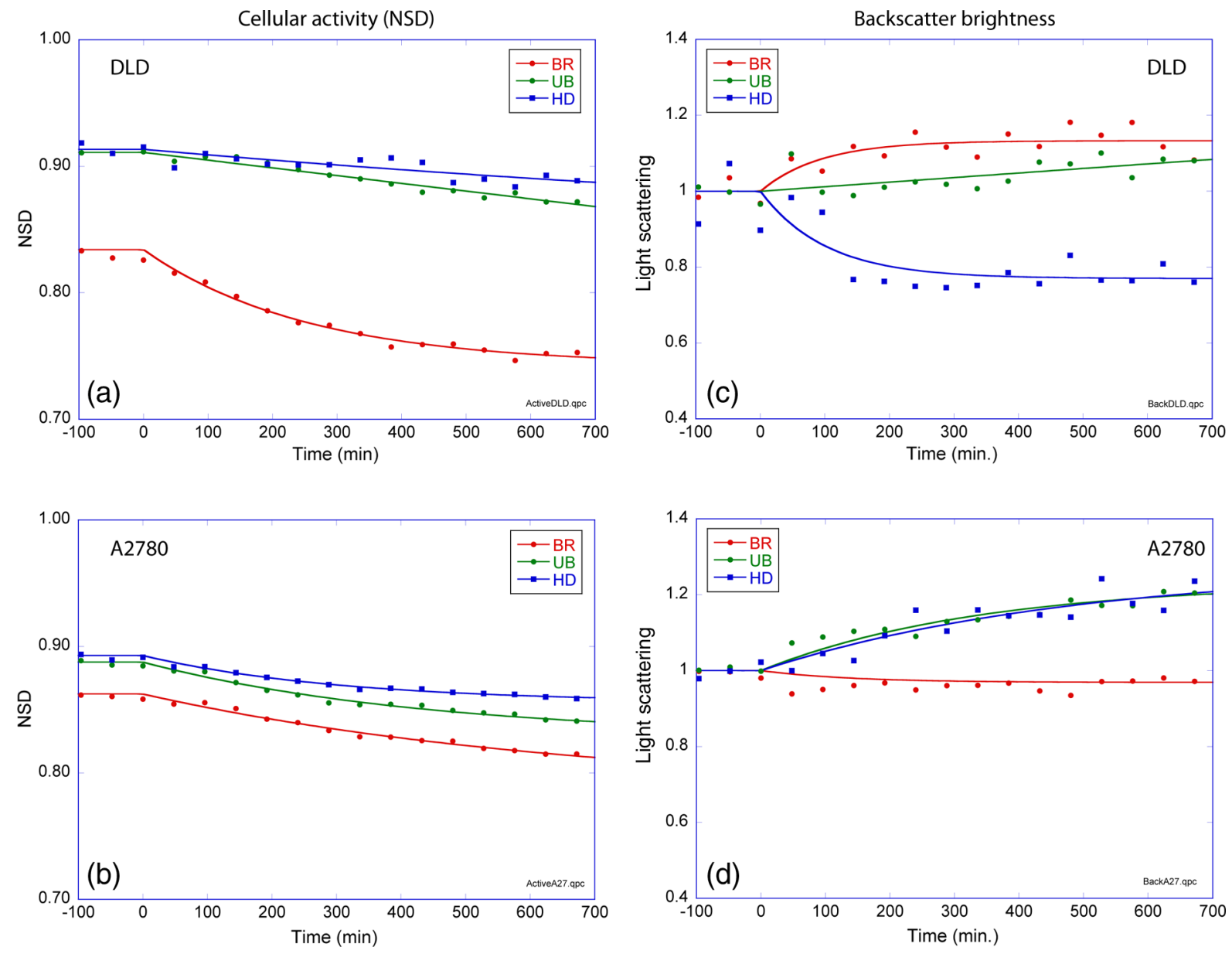

Fig. 7 Average time dependence of the NSD [related to cellular activity through Eq. (3)] and the BB for the three growth techniques (BR, bioreactor; UB, U-bottom; and HD, hanging drop) responding to $10 \mu \mathrm{M}$ paclitaxel. The NSD trends for A2780 and DLD-1 versus time are shown in (a) and (b). The normalized light scattering is shown in (c) and (d). The cellular activity is inhibited most strongly in the BR case, although the light scattering shows no consistent trends for the two cell lines. The average response times to Taxol are $\sim 3 \mathrm{~h}$. 

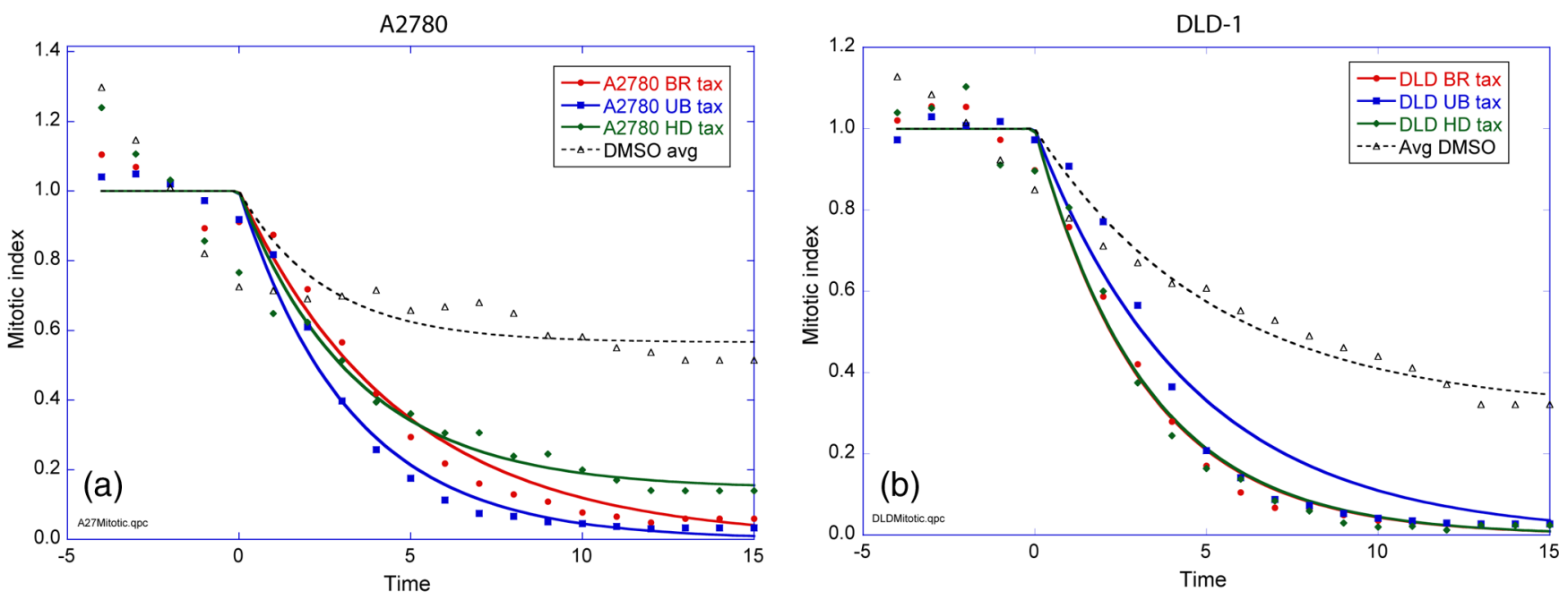

Fig. 8 Mitotic index change caused by the application of $10 \mu \mathrm{M}$ paclitaxel shown as a function of time for (a) A2780 and (b) DLD-1. The response time of $\sim 3 \mathrm{~h}$ for each cell line is almost identical for the three growth methods.

because the drug stabilizes microtubules and prevents mitosis and inhibits cell shape changes. The sample brightness, on the other hand, shows no consistent trend among the cell lines or growth techniques.

BDI can be used to extract direct pharmacodynamic effects by isolating mitotic events inside tissue. Mitotic events are detected in the mid-Doppler frequency band between 0.1 and $1 \mathrm{~Hz}$. Local pixel-based spectrograms are investigated for signatures in this specific spectral range and are scored positive for a mitotic event when the signature occurs within a resolution voxel. To provide sufficient signal-to-noise, the spectra of four pixels are averaged. Because mitosis occurs at a rate of approximately once per day per cell, and each local 4-pixel volume has about 20 cells, there is $\sim 1$ mitotic event per hour in each local spectrogram. Figures 8(a) and 8(b) show the normalized mitotic index change after paclitaxel was applied to tumor spheroids from the two cell lines A2780 and DLD-1 under the different growth approaches. Both cell line types show a pronounced decrease after paclitaxel was applied, compared to the control sample. For both types of spheroids, the response time is around $2.5 \mathrm{~h}$. Interestingly, the mitotic index change is almost identical for each cell line and growth condition. The diffusion rate of paclitaxel into these spheroids takes only minutes because paclitaxel is a small molecule with molecular weight $853.906 \mathrm{~g} / \mathrm{mol}$. The 2-to-3 h response captures the gradual arrest of cell proliferation as cells move through the cell cycle and are arrested at the mitotic checkpoint. The ability of BDI to measure the mitotic index in living tissue responding to cytostatics represents an improvement over available current methods such as long-term growth-delay studies.

\section{Discussion and Conclusions}

BDI can be related to previous work associated with dynamic measurements of cellular processes using coherence-domain techniques that include dynamic light scattering OCT, ${ }^{69}$ fieldbased dynamic light scattering microscopy,${ }^{70}$ diffusion-sensitive $\mathrm{OCT}^{71}{ }^{1}$ and dynamic full-field OCT. ${ }^{72}$ BDI uses coherencedomain detection similar to each of these techniques, but with an emphasis on developing a full speckle field at tissue scales that generates a high dynamic range with high signal-to-noise per voxel. This makes it possible to differentiate subtle differences in the action of xenobiotics on tissue activity. In addition, BDI penetrates deeply into dense tissue up to 1-mm deep because imaging aberrations do not degrade its $20-\mu \mathrm{m}$ resolution. The tradeoff between resolution and dynamic range has established a compromise at this resolution scale that is also compatible with off-axis holography that might limit higher-resolution approaches. The BDI system used in this work had a MachZender configuration and was not common path (which has the advantage of low sensitivity to mechanical vibrations ${ }^{73}$ ), but the system has passive vibration isolation that removes vibration background. In addition, the digital image reconstruction takes the modulus of the Fourier-reconstructed image and does not use the complex values, which makes phase stability unnecessary for this implementation.

The ability to monitor a living sample nondestructively over time opens an avenue for longitudinal drug exposure studies. This paper focused on the use of BDI for the assessment of physiological and pharmacological properties of 3-D tissues grown using different culture methods. Use of 3-D tissue is a new trend in cancer drug screening, but there has been a proliferation of different methods to grow 3-D tissues with a lack of complete characterization of the different drug responses. Because drug screening is already fraught with inconsistencies leading to difficulties moving promising new compound leads into approved drugs, it is critically important to establish the relevant tissue properties that best recapitulate the targeted in vivo behavior. Therefore, this paper focused on the development of BDI as a new tool for assessment of drug response in various types of 3-D tissue, including in-vitro and ex-vivo models.

The four growth techniques considered in this paper differ in time and manner of growth. The nonadherent UB plates have become one of the easiest means to generate tumor spheroids in only a matter of two or three days, and hence has gained popularity, but the rapid growth provides no time for the development of tight tissue structure or the generation of ECM, which can be an important factor in inter- and intracellular signaling. The HD technique is similar to the UB approach, but produces more homogeneous behavior of the samples in terms of their biodynamic signatures. The BR growth is notably different 
from these other two tissue culture methods, taking up to 1 month to grow a batch of tumor spheroids. The slow growth provides sufficient time for BR-grown spheroids to develop traces of ECM and also tighter tissue structures. The tighter tissue structures present low diffusion coefficients for oxygen and nutrients and hinders the out diffusion of metabolites, creating hypoxic cores for moderate-sized tumor spheroids and even necrotic cores for spheroids with diameters larger than $500 \mu \mathrm{m}$. The BR-grown cultured spheroids are most similar to biopsies in their biodynamic signatures, including the wide heterogeneity of tissue biodynamic properties.

\section{Disclosures}

David Nolte and John Turek have a financial interest in Animated Dynamics Inc., which is licensing biodynamic imaging technology from the Office of Technology Commercialization of Purdue University.

\section{Acknowledgments}

This work was supported by grants NSF 1263753-CBET and NIH NIBIB 1RO1EB016582-02. We also thank 3-D Biomatrix for supplying the materials to perform the hanging-drop growth.

\section{References}

1. C. R. Thoma et al., "3D cell culture systems modeling tumor growth determinants in cancer target discovery," Adv. Drug Delivery Rev. 69, 29-41 (2014).

2. R. Straussman et al., "Tumour micro-environment elicits innate resistance to RAF inhibitors through HGF secretion," Nature 487, 500-504 (2012).

3. S. Breslin and L. O'Driscoll, "Three-dimensional cell culture: the missing link in drug discovery," Drug Discovery Today 18(5-6), 240-249 (2013).

4. E. Fennema et al., "Spheroid culture as a tool for creating 3D complex tissues," Trends Biotechnol. 31, 108-115 (2013).

5. M. Rimann and U. Graf-Hausner, "Synthetic 3D multicellular systems for drug development," Curr. Opin. Biotechnol. 23, 803-809 (2012).

6. S. J. Smith et al., "Recapitulation of tumor heterogeneity and molecular signatures in a 3D brain cancer model with decreased sensitivity to histone deacetylase inhibition," Plos One 7(12), e52335 (2012).

7. X. Xu, M. C. Farach-Carson, and X. Q. Jia, "Three-dimensional in vitro tumor models for cancer research and drug evaluation," Biotechnol. Adv. 32, 1256-1268 (2014).

8. L. B. Weiswald, D. Bellet, and V. Dangles-Marie, "Spherical cancer models in tumor biology," Neoplasia 17, 1-15 (2015).

9. M. Rimann et al., "An in vitro osteosarcoma 3D microtissue model for drug development," J. Biotechnol. 189, 129-135 (2014).

10. R. H. Webb, "Confocal optical microscopy," Rep. Progr. Phys. 59, 427-471 (1996)

11. J. Huisken et al., "Optical sectioning deep inside live embryos by selective plane illumination microscopy," Science 305, 1007-1009 (2004).

12. J. M. Schmitt, "Optical coherence tomography (OCT): a review," IEEE J. Sel. Top. Quantum Electron. 5, 1205-1215 (1999).

13. W. Drexler, "Ultrahigh-resolution optical coherence tomography," J. Biomed. Opt. 9, 47-74 (2004).

14. C. L. Evans et al., "In vitro ovarian tumor growth and treatment response dynamics visualized with time-lapse OCT imaging," Opt. Express 17, 8892-8906 (2009).

15. O. J. Klein, Y. K. Jung, and C. L. Evans, "Longitudinal, quantitative monitoring of therapeutic response in 3D in vitro tumor models with OCT for high-content therapeutic screening," Methods 66, 299-311 (2014).

16. Y. Jung et al., "Label-free, longitudinal visualization of PDT response in vitro with optical coherence tomography," Isr. J. Chem. 52, 728-744 (2012).

17. C. L. Evans et al., "Killing hypoxic cell populations in a $3 \mathrm{D}$ tumor model with EtNBS-PDT," Plos One 6(8), e23434 (2011).
18. D. D. Nolte et al., "Holographic tissue dynamics spectroscopy," J. Biomed. Opt. 16, 087004 (2011).

19. K. Jeong, J. J. Turek, and D. D. Nolte, "Speckle fluctuation spectroscopy of intracellular motion in living tissue using coherence-domain digital holography," J. Biomed. Opt. 15, 030514 (2010).

20. K. Jeong, J. J. Turek, and D. D. Nolte, "Imaging motility contrast in digital holography of tissue response to cytoskeletal anti-cancer drugs," Opt. Express 15, 14057-14064 (2007).

21. Z. Yaqoob et al., "Homodyne en face optical coherence tomography," Opt. Lett. 31, 1815-1817 (2006).

22. I. Yamaguchi and T. Zhang, "Phase-shifting digital holography," Opt. Lett. 22, 1268-1270 (1997).

23. E. Cuche, F. Bevilacqua, and C. Depeursinge, "Digital holography for quantitative phase-contrast imaging," Opt. Lett. 24, 291-293 (1999).

24. T. Zhang and I. Yamaguchi, "Three-dimensional microscopy with phase-shifting digital holography," Opt. Lett. 23, 1221-1223 (1998).

25. K. K. Bizheva, A. M. Siegel, and D. A. Boas, "Path-length-resolved dynamic light scattering in highly scattering random media: The transition to diffusing wave spectroscopy," Phys. Rev. E 58, 7664-7667 (1998).

26. A. Wax et al., "Path-length-resolved dynamic light scattering: modeling the transition from single to diffusive scattering," Appl. Opt. 40, 42224227 (2001).

27. J. Evans et al., "Fluctuations of the red blood cell membrane: relation to mechanical properties and lack of ATP dependence," Biophys. J. 94, 4134-4144 (2008).

28. H. Strey and M. Peterson, "Measurement of erythrocyte-membrane elasticity by flicker eigenmode decomposition," Biophys. J. 69, 478-488 (1995).

29. K. Tamura et al., "Active fluctuation in the cortical cytoskeleton observed by high-speed live-cell scanning probe microscopy," Acta Biomater. 7, 3766-3772 (2011).

30. T. Betz et al., "ATP-dependent mechanics of red blood cells," Proc. Natl. Acad. Sci. U. S. A. 106, 15320-15325 (2009).

31. X. L. Nan, P. A. Sims, and X. S. Xie, "Organelle tracking in a living cell with microsecond time resolution and nanometer spatial precision," Chem. Phys. 9, 707-712 (2008).

32. V. Racine et al., "Visualization and quantification of vesicle trafficking on a three-dimensional cytoskeleton network in living cells," J. Microsc. Oxford 225, 214-228 (2007).

33. N. A. Brazhe et al., "Unraveling cell processes: interference imaging interwoven with data analysis," J. Biol. Phys. 32, 191-208 (2006).

34. B. Trinczek, A. Ebneth, and E. Mandelkow, "Tau regulates the attachment/detachment but not the speed of motors in microtubule-dependent transport of single vesicles and organelles," J. Cell. Sci. 112, 2355-2367 (1999).

35. D. D. Nolte et al., "Tissue dynamics spectroscopy for phenotypic profiling of drug effects in three-dimensional culture," Biomed. Opt. Express 3, 2825-2841 (2012).

36. J. A. Lee and E. L. Berg, "Neoclassic drug discovery: the case for lead generation using phenotypic and functional approaches," J. Biomol. Screen. 18, 1143-1155 (2013).

37. N. T. Shaked et al., "Parallel on-axis holographic phase microscopy of biological cells and unicellular microorganism dynamics," Appl. Opt. 49, 2872-2878 (2010).

38. B. Marrero, J. L. Messina, and R. Heller, "Generation of a tumor spheroid in a microgravity environment as a $3 \mathrm{D}$ model of melanoma," In Vitro Cell. Dev. Biol. Animal 45, 523-534 (2009).

39. T. T. Chang and M. Hughes-Fulford, "Monolayer and spheroid culture of human liver hepatocellular carcinoma cell line cells demonstrate distinct global gene expression patterns and functional phenotypes," Tissue Eng. Part A 15, 559-567 (2009).

40. P. Yu et al., "Holographic optical coherence imaging of rat osteogenic sarcoma tumor spheroids," Appl. Opt. 43, $4862-4873$ (2004).

41. O. Sirenko et al., "High-content assays for characterizing the viability and morphology of 3D cancer spheroid cultures," Assay Drug Dev. Technol. 13, 402-414 (2015).

42. J. E. Ekert et al., "Three-dimensional lung tumor microenvironment modulates therapeutic compound responsiveness in vitro-implication for drug development," Plos One 9(3), e92248 (2014).

43. A. I. Neto et al., "A novel hanging spherical drop system for the generation of cellular spheroids and high throughput combinatorial drug screening," Biomater. Sci. 3, 581-585 (2015). 
44. S. Raghavan et al., "Formation of stable small cell number threedimensional ovarian cancer spheroids using hanging drop arrays for preclinical drug sensitivity assays," Gynecol. Oncol. 138, 181-189 (2015).

45. D. Yip and C. H. Cho, "A multicellular 3D heterospheroid model of liver tumor and stromal cells in collagen gel for anti-cancer drug testing," Biochem. Biophys. Res. Commun. 433, 327-332 (2013).

46. J. M. Kelm et al., "Method for generation of homogeneous multicellular tumor spheroids applicable to a wide variety of cell types," Biotechnol. Bioeng. 83, 173-180 (2003).

47. K. O. Hicks et al., "Use of three-dimensional tissue cultures to model extravascular transport and predict in vivo activity of hypoxia-targeted anticancer drugs," J. Nat. Cancer Inst. 98, 1118-1128 (2006).

48. J. K. Tunggal et al., "Penetration of anticancer drugs through solid tissue: a factor that limits the effectiveness of chemotherapy for solid tumors," Clin. Cancer Res. 5, 1583-1586 (1999).

49. R. Grantab, S. Sivananthan, and I. F. Tannock, "The penetration of anticancer drugs through tumor tissue as a function of cellular adhesion and packing density of tumor cells," Cancer Res. 66, 1033-1039 (2006).

50. M. Leushacke and N. Barker, "Ex vivo culture of the intestinal epithelium: strategies and applications," Gut 63, 1345-1354 (2014).

51. X. N. Li et al., "Oncogenic transformation of diverse gastrointestinal tissues in primary organoid culture," Nat. Med. 20, 769-777 (2014).

52. L. Vesci et al., "Trastuzumab and docetaxel in a preclinical organotypic breast cancer model using tissue slices from mammary fat pad: translational relevance," Oncol. Rep. 34, 1146-1152 (2015).

53. K. A. T. Naipal et al., "Tumor slice culture system to assess drug response of primary breast cancer," BMC Cancer 16, 78 (2016).

54. J. Bereiter-Hahn, "Mechanics of crawling cells," Med. Eng. Phys. 27, 743-753 (2005).

55. R. D. Eppinga et al., "Tropomyosin and caldesmon regulate cytokinesis speed and membrane stability during cell division," Arch. Biochem. Biophys. 456, 161-174 (2006).

56. L. Norman, K. Sengupta, and H. Aranda-Espinoza, "Blebbing dynamics during endothelial cell spreading," Eur. J. Cell Biol. 90, 37-48 (2011).

57. G. Farhat et al., "Detecting apoptosis using dynamic light scattering with optical coherence tomography," J. Biomed. Opt. 16, 070505 (2011).

58. M. Suissa et al., "Internal dynamics of a living cell nucleus investigated by dynamic light scattering," Eur. Phys. J. E 26, 435-448 (2008).

59. M. J. Schnitzer and S. M. Block, "Kinesin hydrolyses one ATP per 8-nm step," Nature 388, 386-390 (1997).

60. A. D. Mehta et al., "Myosin-V is a processive actin-based motor," Nature 400, 590-593 (1999).

61. A. S. Robertson, E. Smythe, and K. R. Ayscough, "Functions of actin in endocytosis," Cell. Mol. Life Sci. 66, 2049-2065 (2009).

62. D. A. Boas and A. K. Dunn, "Laser speckle contrast imaging in biomedical optics," J. Biomed. Opt. 15, 011109 (2010).

63. J. Parness and S. B. Horwitz, "Taxol binds to polymerized tubulin in vitro," J. Cell Biol. 91, 479-487 (1981).

64. D. Merrill et al., "Digital holography of intracellular dynamics to probe tissue physiology," Appl. Opt. 54, A89-A97 (2015).

65. I. F. Tannock et al., "Limited penetration of anticancer drugs through tumor tissue: a potential cause of resistance of solid tumors to chemotherapy," Clin. Cancer Res. 8, 878-884 (2002).

66. F. B. Pruijn et al., "Prediction of tumour tissue diffusion coefficients of hypoxia-activated prodrugs from physicochemical parameters," Aust. J. Chem. 61, 687-693 (2008).

67. A. M. Al-Abd et al., "Novel application of multicellular layers culture for in situ evaluation of cytotoxicity and penetration of paclitaxel," Cancer Sci. 99, 423-431 (2008).
68. A. M. C. Yvon, P. Wadsworth, and M. A. Jordan, "Taxol suppresses dynamics of individual microtubules in living human tumor cells," Mol. Biol. Cell 10, 947-959 (1999).

69. J. Lee et al., "Dynamic light scattering optical coherence tomography," Opt. Express 20, 22262-22277 (2012).

70. C. Joo and J. F. de Boer, "Field-based dynamic light scattering microscopy: theory and numerical analysis," Appl. Opt. 52, 7618-7628 (2013).

71. R. L. Blackmon et al., "Imaging extracellular matrix remodeling in vitro by diffusion-sensitive optical coherence tomography," Biophys. J. 110, 1858-1868 (2016).

72. C. Apelian et al., "Dynamic full field optical coherence tomography: subcellular metabolic contrast revealed in tissues by interferometric signals temporal analysis," Biomed. Opt. Express 7, 1511-1524 (2016).

73. Y. Zhu et al., "Spectral-domain differential interference contrast microscopy," Opt. Lett. 36, 430-432 (2011).

Hao Sun received his $\mathrm{PhD}$ in physics from Purdue University in 2016. He studied dynamic holography in both semiconductors and biomedical optics under the direction of Dr. David Nolte. He currently works on mobile search ads for cars at Google, Mountain View, California, USA.

Daniel Merrill received his PhD from Purdue University in 2016. He completed his doctoral studies under the direction of Dr. David Nolte with whom he developed biodynamic imaging into a predictive assay for patient therapy selection. He currently teaches physics at Indiana State University where he is developing undergraduate laboratory courses that integrate cross-disciplinary experimental design.

Ran An received his BS degree from the University of Science and Technology, China, in 2008, and his PhD from the Department of Physics and Astronomy at Purdue University in 2014, developing the science and technology of biodynamic imaging. He is a technical founder and the chief technology officer of Animated Dynamics, Inc., a biotech start-up located in Indianapolis that is commercializing biodynamic imaging technology and services.

John Turek received his PhD from the University of Illinois at Urbana in Veterinary Medical Science. He is a professor in the Department of Basic Medical Sciences at Purdue University College of Veterinary Medicine. For the past 16 years, his research has been in the area of biodynamic imaging with a focus on the use of 3-D cell cultures and BDI for derisking drug discovery and for cancer chemotherapy selection.

Daniela Matei received her MD from the University of Medicine and Pharmacy "Carol Davila" and completed postgraduate training at SUNY at Stony Brook and at UCLA. She is a professor in the Department of Obstetrics and Gynecologyoined at Northwestern University Feinberg School of Medicine specializing in ovarian cancer. Her laboratory studies mechanisms of metastasis and therapeutics. She is engaged in clinical research testing therapies for ovarian cancer.

David D. Nolte received his BS degree from Cornell University in 1981 and his PhD from the University of California at Berkeley in 1988, followed by a postdoctoral appointment at AT\&T Bell Labs. $\mathrm{He}$ is the E.M Purcell distinguished professor of physics at Purdue University. He has been elected as a fellow of the Optical Society of America, a fellow of the American Physical Society, and a fellow of the AAAS. He is the technical founder of two biotech startup companies. 